Erschienen in: Klein, Josef (Hrsg.): Politische Semantik. Bedeutungsanalytische und sprachkritische

Beiträge zur politischen Sprachverwendung. - Opladen: Westdeutscher Verlag, 1989. S. 153-186. DOI: https://doi.org/10.1007/978-3-322-91068-4_4

\title{
INTERESSENABHÄNGIGER UMGANG MIT WÖRTERN IN DER UMWELTDISKUSSION
}

\author{
Ulrike $\mathrm{Ha \beta}$
}

1. Was ist Umweltdiskussion?

2. Was ist Umweltwortschatz?

3. Öfentliche und linguistische Sprachkritik am Umweltwortschatz

4. Bezeichnungsvarianten in Expertentexten

5. Bezeichnungsvarianten in Umweltlexika

5.1 Stichwörter, Verweise und sog. Synonyme

5.2 Stichwortfelder

5.3 Müll oder Abfall?

5.4 Atomkraft oder Kernenergie? (mit einem historischen Exkurs)

5.5 Sachorientierte und problemorientierte Darstellung

6. Zusammenfassung

7. Anhang: Zwei Tabellen

8. Quellen

9. Literatur

0. In diesem Beitrag möchte ich versuchen darzustellen, wie sich die gegensätzlichen gesellschaftlichen Positionen in bezug auf zentrale Umweltthemen in populären Nachschlagewerken niederschlagen. Dabei konzentriere ich mich auf die Bezeichnungsvarianten in den Feldern Giftmüll/Problemabfall und Atomkraft,-energie/Kernkraft, -energie und darauf, wie sie von den Lexikonautoren behandelt werden. Den impliziten und expliziten Bewertungen von Wörtern in den Lexika wird die (tatsächliche) Wortverwendung in den öffentlichen (Massen-)Medien gegenübergestellt.

\section{Was ist Umweltdiskussion?}

Eine Untersuchung bestimmter Wortfelder und einzelner, wenn auch repräsentativer Wörter, wie ich sie in diesem Beitrag vornehmen will, muß, wenn sie eine linguistisch-pragmatische und handlungssemantische Untersuchung sein soll, zunächst den Rahmen des handelnden Sprechers bestimmen, aus dem heraus das je Besondere der einzelnen Wörter erklärt werden kann. 
Ich will Umweltdiskussion auffassen als Teil der öffentlichen Diskussion insgesamt. Sie findet in und mit Texten statt, mündlichen und vor allem schriftlichen Texten, die öffentlich, d.h. prinzipiell für jeden zugänglich sind. Dazu zählen die Texte in Rundfunk, Fernsehen, allgemeinen Zeitungen, speziellen Zeitschriften und in Büchern, vor allem in solchen, die für ein breites Publikum, nicht ausschließlich für Fachleute geschrieben sind.

Texte, die ich zur Umweltdiskussion zähle, sind außer durch Öffentlichkeit durch ihren Bezug auf das Thema Umwelt oder auf ein Teilthema dessen gekennzeichnet. Das Besondere an diesem Thema ist seine Karriere, die es in den vergangenen zwanzig Jahren von den journalistischen Ressorts "Aus aller Welt", "Vermischtes" u.ä. über die wissenschaftsjournalistischen Rubriken "Aus Forschung und Technik" u.ä. auf die politischen Seiten und bis in die Leitartikel katapultiert hat. Diese zunehmende Politisierung des Themas Umwelt allein ließe schon, wenn man es aus eigener Anschauung noch nicht wüßte, eine Interessengebundenheit im Gebrauch sprachlicher Ausdrucksmittel vermuten.

Die Menge der die Umweltdiskussion bildenden Texte läßt sich nach ihrer Zugehörigkeit zu einer Reihe von Textsorten klassifizieren. Textsorten fasse ich hier so auf, wie sie von den Sprachteilhabern selbst, nicht von Linguisten im Rahmen einer Texttypologie, identifiziert und unterschieden werden ${ }^{1}$; es kommt mir dabei auf die Erwartungen bzw. Vorannahmen an, die beispielsweise mit einer Textsorte wie der der später zu behandelnden Umweltlexika verbunden sind.

Es kommt mir außerdem darauf an, daß verschiedene Textsorten untereinander in einer Weise zusammenhängen, die Einfluß auf die Verwendungsbedingungen von Wörtern hat.

Einen solchen Zusammenhang sehe ich in den von Textsorte zu Textsorte fortschreitenden Popularisierungsprozessen ${ }^{2}$, bei denen ursprüngliche Expertentexte der fachinternen Kommunikation zu Texten der Laieninformation, zu Anweisungs-, aber auch zu Werbe- und Überzeugungstexten transformiert werden. Dieses vollzieht sich meistens über mehrere Stufen. Während des Popularisierungsprozesses können sich kommunikative Absichten und andere Textcharakteri-

Vgl. E. Gülich 1986

2

Das Prinzip solcher Prozesse habe ich an einem historischen Beispiel erläutert in $\mathrm{HaB}$ 1986. 
verhalte handelt, weitgehend unverändert. In Texten am anderen Ende der Popularisierungsskala tauchen damit Termini technici auf, von denen zunächst unklar ist, ob sie ihre alte, fachsprachlich bedingte Wertungsneutralität, die "Appellfunktion Null", wie Hermanns (1986, S.158) sagt, noch besitzen oder durch den anderen, politischeren Kommunikationsrahmen zwangsläufig verloren haben und welcher Art ihre Parteilichkeit und Appellfunktionen dann sind.

\section{Was ist Umweltwortschatz?}

Umweltwortschatz, verstanden als Wortschatz aller der Texte, die die Umweltdiskussion ausmachen, kann nicht der Wortschatz eines bestimmten Subsystems sein, wie ihn die Fachsprachenlinguistik auffaßt und beschrieben hat. ${ }^{3}$ Vielmehr gehört er zum Diasystem der Allgemeinsprache der Gegenwart, zur Sprache der öffentlichen und politischen Kommunikation und trägt dabei nicht wenig zum Eindruck ihrer manchmal beklagten "Verwissenschaftlichung" bei. Somit gelten für die Wörter der Umweltdiskussion uneingeschränkt dieselben kommunikativen Bedingungen wie für andere Wörter in öffentlichen und politischen Auseinandersetzungen.

Dennoch halte ich diesen Wortschatzbereich aufgrund besonderer Merkmale für hinreichend abgrenzbar; diese Merkmale seien im Folgenden (2.1 bis 2.4) $\mathrm{kurz}^{4}$ skizziert.

2.1 Diachron gesehen lassen sich mehrere Fach- bzw. Wissenschaftssprachen als Quellen von Umweltwörtern ausmachen. Der Bereich der Technik und der industriellen Produktion fällt hier quantitativ viel stärker ins Gewicht als der Bereich der wissenschaftlichen Ökologie, Biologie und anderer Naturwissenschaften. Einen ebenfalls sehr hohen Anteil hat der Wortschatz der Verwaltung und kommunalen Organisation und, in zunehmendem Maße, der juristische Wortschatz aus der Umweltgesetzgebung. Insgesamt ist der Anteil im herkömmlichen Sinn aufgefaßter "Fremdwörter" aus dem Griechisch-

3

Resümierend z.B. Möhn / Pelka 1984.

4

Eine ausführlichere Beschreibung des Wortschatzes der Umweltdiskussion entsteht zu Zeit im Rahmen des "Lexikons schwerer Wörter im Deutschen" am Institut für deutsche Sprache (Mannheim), das 1989 erscheinen soll. 
Lateinischen und aus dem Englischen relativ gering ${ }^{5}$, der Anteil schwer verständlicher "deutscher" Wörter, wie sie von Juristen und Bürokraten geprägt werden, dagegen recht umfangreich.

2.2 Je mehr Lebens- und Gesellschaftsbereiche das Thema Umwelt durchdringt, desto größer scheint die Menge der "neuen" Wörter zu werden, die aus produktiven Elementen (z.B. Öko-, Bio-, Umwelt-, Atom-, Recycling-) gebildet werden.

Diese außerordentliche Produktivität ist ein weiteres, ausdrucksseitiges Charakteristikum des Umweltwortschatzes, auf dessen generelle semantische Aspekte ich in diesem Rahmen nicht eingehen kann.

2.3 Ein Aspekt der Wortbildungsproduktivität besteht darin, daß die Entstehung von scheinbar synonymen Ausdrücken wie Atomkraft und Kernkraft begünstigt wird, die ich im folgenden Bezeichnungsvarianten (oder Konkurrenzausdrücke) nennen will.

Linguistisch gesehen fällt zwar die prädizierende (charakterisierende) Funktion dieser Ausdrücke neben ihrer referentiellen (bezeichnenden) Funktion außerordentlich stark ins Gewicht; den Sprechern hingegen geht es dabei, je nach Standpunkt, um 'Richtigkeit' und 'Angemessenheit' in bezug auf die zu bezeichnende Sache: Entsorgungspark oder Plutoniumfabrik, Problemfall oder Giftmüll, (Super-)GAU oder Reaktorkatastrophe. Innerhalb der Umweltdiskussion konkurrieren diese Ausdrücke miteinander, z.T. so stark, daß sie zu Fahnen- und Stigmawörtern ${ }^{6}$ der gegnerischen Gruppierungen werden. Das Charakteristikum konkurrierender Varianten hat der Umweltwortschatz mit politischem Wortschatz gemein. ${ }^{\mathbf{7}}$

2.4 Als letztes Charakteristikum sei die deutliche Tendenz zu metaphorischen Verwendungen fach- und sachbezogener Ausdrücke genannt. Zwei Arten oder Stufen von Metaphorisierungen lassen sich

5 Genau genommen gehören die Bezeichnungen für chemische Substanzen und für Maßeinheiten wie z.B. 'FCKW', 'Dioxin', 'Bequerel' auch zum Umweltwortschatz; sie sollen hier aber ausgeklammert werden, weil sie semantisch auch in fachexternen Kontexten absolut scharf und fixiert sind und ihnen somit wesentliche Merkmale gemeinschaftlicher Lexik fehlen.

6 Zur Kennzeichnung besonderer Appellativität hat Hermanns 1980 die Ausdrücke 'Fahnenwort' und 'Stigmawort' eingeführt.

Vgl. Strauß / Zinfonun 1985, S. 100 - 103: 'wertende[r] und manipulativ eingesetzte[r] konkurrierende[r] "Doppelbenennungen"' und Strauß 1986, S. 118ff., wo "ideologische Bezeichnungsvarianten" im politischen Wortschatz behandelt werden. 
erkennen. Die erste findet innerhalb desselben engeren Themenbereichs statt, in dem auch die nicht-metaphorische Verwendung eines umwelttypischen Ausdrucks vorkommt. Typisch hierfür ist der folgende Fall: Im Zusammenhang mit der Berichterstattung über einen Störfall im Hochtemperaturreaktor Hamm äußert ein Journalist im Mannheimer Morgen vom 3.6.1986 Kritik an der Informationspraxis des zuständigen Ministers mit der metaphorischen Titelzeile "Informations-Störfall. Wie man der Bevölkerung Angst macht". Von "Informations-Gau" wurde schon nach Tschernobyl wiederholt gesprochen. Kritik am (umwelt-)politischen Handeln des Gegners ist ein wesentliches Motiv für die metaphorische "Entwendung" eines meistens aus dem technischen oder administrativen Bereich herkommenden - Wortes.

Für die zweite, bereichsexterne Metaphorisierung lieferte der Historikerstreit im Sommer 1986 das schon klassisch zu nennende Beispiel "Entsorgung der Vergangenheit". Das Paradigma wurde aufgegriffen in der Auseinandersetzung um die Ausgrabungsfunde des früheren jüdischen Gettos am Frankfurter Börneplatz: ein Graffitto dort lautete "Restrisiko Geschichte" (Frankfurter Rundschau 7.9.1987). Bereichsexterne Metaphorisierungen von Umweltwörtern sind ansonsten bei Glossenschreibern, Kabarettisten und anderen sehr beliebt - eine Entwendung ins Unernste?

Mit dieser notwendigerweise unzureichenden Skizzierung einiger Charakteristika des Umweltwortschatzes, die lediglich als Orientierungsrahmen für das Folgende dienen soll, will ich es bewenden lassen.

Die relativ hohe und durchweg kritische Aufmerksamkeit, die Wörter und formelhafte Wendungen der Umweltdiskussion in der Öffentlichkeit auf sich ziehen, kann letztlich ebenfalls zu den charakteristischen Eigenheiten gezählt werden, die die Verwendungsbedingungen und damit die Bedeutung von Wörtern in bestimmter Weise prägen.

3. Öffentliche und linguistische Sprachkritik am Umweltwortschatz

Das Bewußtsein, daß der Umgang mit Ausdrücken und Ausdrucksweisen in der Umweltdiskussion interessenabhängig ist, war zu allererst kein linguistisches oder ein durch die Arbeit von Linguisten her- 
vorgebrachtes Bewußtsein, sondern wir verdanken es einer allgemeinen und öffentlich artikulierten skeptischen Hellhörigkeit. Es artikulierte sich zum ersten Mal unüberhörbar am Fall des Entsorgungsparks. Hier stand einem einzigen denotierenden Verwendungsfall in einem Artikel der WELT vom 22.1.1976 eine Vielzahl von zitierenden, distanzierenden, ironischen Äußerungen, vor allem in Glossen, gegenüber. ${ }^{8}$ In den Sprachkritiken zum Entsorgungspark verdichtete sich nun eine Meinung zum Topos, nämlich die, daß Politiker mittels gesuchter Bezeichnungen schlimme Sachverhalte verschleiern, verschönern und den Bürger verdummen wollten; diese Auffassung wurde zu einem festen Bestandteil des allgemeinen Sprachbewußtseins in bezug auf die sogenannte Umwelt- oder Ökö-Sprache. Der Fall Entsorgungspark lieferte von da ab das Funktionsmuster für die öffentliche Auseinandersetzung mit weiteren sprachlichen Ausdrücken im Bereich des Themas Umwelt.

Bevor ich im folgenden die - mögliche - Interessengebundenheit konkurrierender Ausdrücke vom Typ Abfall/Müll und Atomkraft/ Kernkraft aus linguistischer Perspektive untersuche, möchte ich kurz zeigen, welche Argumentationen in der öffentlichen oder in der "Sprachkritik von unten"9 vorherrschen und auf welchen Vorannahmen hinsichtlich des Verhältnisses von Wörtern und Sachverhalten diese Kritik beruht.

Zu dem Zweck habe ich eine Sammlung von Zeitungsartikeln und Glossen $^{10}$ zum Thema Ökö-Sprache zwischen Sommer 1983 und Sommer 1985 daraufhin durchgesehen, welches die Zielpunkte der Kritik sind und womit die begründet werden. Dabei fand sich, daß einige Kritikpunkte stereotyp wiederholt werden.

Erstens die alte und ewige Fremdwörterklage, jetzt auch in bezug auf chemische Stoffbezeichnungen, allerdings in der Variante, daß niemand mehr ernsthaft eine "Übersetzung" von polychlorierte Biphenyle verlangt, denn bei den Umwelt-Fremdwörtern wird endlich of fenkundig, daß man sich mit Verdeutschungen wie Dünnsäure oder Berliner Blau lediglich die viel schlimmeren Euphemismen einhandelt.

8 Tatsächlich mag es einige primär denotierende Verwendungen in der Öfentlichkeit mehr gegeben haben, mir ist ein zweiter Beleg jedoch nie bekannt geworden.

9

Diesen Ausdruck verdanke ich R. Wimmer.

10

Ich danke K. Plastwich von der Dokumentationsstelle des IDS. 
Zweitens werden Schlag- und Modewörter wie bio-, öko-, freundlich und Umwelt- gegeißelt, teils mit der Begründung, inhaltsleer und nichtssagend zu sein, teils mit der Feststellung, die Wörter stimmten nicht mit den Inhalten überein und dienten der Vorspiegelung falscher Tatsachen.

Drittens werden die recht zahlreichen Bürokratismen, besonders im Abfallrecht (Wertstoff, Reststoff, Abprodukt, Sondermüllkleinmengenentsorgung), auf's Korn genommen. Dabei wird von "Wortblüten" und "Sprachregelungen" gesprochen, ohne daß die Frage nach der konkreten Interessengebundenheit solcher Regelungen, nämlich: Wer regelt mit dem Ausdruck $\mathrm{X}$ wem gegenüber was in welcher Absicht?, gestellt würde.

Viertens: Die kritische Feststellung, daß namentlich "Politiker" oder auch "die Medien" Wörter manipulativ einsetzen, ist allgemein, wird im Grunde immer schon vorausgesetzt und gipfelt im zentralen Punkt aller Öko-Sprachkritik, dem Euphemismus-Vorwurf: Mit Wörtern wird verschleiert, geschönt und getäuscht. Begründet wird die Feststellung, daß es sich bei einem bestimmten Ausdruck um einen Euphemismus handelt, mit den Assoziationen, die der Ausdruck beim Sprecher hervorruft, z.B. daß Kernkraft die Assoziation 'kernig, gesund' hervorruft. Dabei setzt jeder Assoziierer voraus, daß Asso-ziationen auch die aller anderen Sprachteilhaber sind, was offensichtlich nicht stimmt.

Es stimmt immer dann nicht, wenn mindestens zwei Ausdrücke als Bezeichnungen inbezug auf (scheinbar) denselben Sachverhalt miteinander konkurrieren. Welche von zweien jeweils als euphemistisch oder 'falsch' und welche als 'richtige' bzw. wertneutrale eingestuft wird, hängt von der politisch-weltanschaulichen Einstellung der Sprecher ab, z.B. Entsorgungspark oder neuerdings: Entsorgungszentrum oder Plutoniumfabrik. Offensichtlich dreht sich die Kontroverse hier nicht mehr um die "richtigere" Bezeichnung für einen eindeutigen Sachverhalt, sondern um die Auffassung dieses Sachverhalts selbst.

Im Bereich des politischen Wortschatzes hat die Konkurrenz von Ausdrücken wie Berufsverbot und Radikalenerla $\beta$ vor über 10 Jahren diese Strittigkeit deutlich gemacht; im Bereich des Umweltwortschatzes versuchen derzeit einige Sprechergruppen, ihre jeweiligen Sachverhaltsperspektiven und, z.T. assoziativ "begründeten", Bewertungen wie bei Kernkraft vs. Atomkraft oder Giftmüll vs. Problem- oder Sonderabfall auf verschiedenen Wegen durchzusetzen. 
Die genannten Glossen oder Zeitungsartikel stellen einen dieser Wege dar. Es gibt aber auch noch andere Wege in Form anderer Textsorten, um konkurrierende Ausdrücke in ein Schema von Positiv-negativ-Bewertungen und Interessengebundenheit einzuordnen und dieses Schema möglichst fest als verbindlich in der öffentlichen Meinung zu verankern.

Bezüglich der öffentlichen Öko-Sprachkritik in Zeitungsartikeln möchte ich folgenden linguistischen Schluß ziehen: In bzw. mit diesen Texten entstand das Bewußtsein der Interessengebundenheit von Ausdrücken, wahrscheinlich artikulierte es sich dort zeitlich auch am frühesten. Aber in diesen Texten wird die Zuordnung von Ausdrükken zu bestimmten Interessen, politischen Absichten und gesellschaftlichen Gruppen auch geschaffen. Natürlich wird sie nicht nur dort geschaffen, aber die wiederholt explizit gemachten Assoziationen fixieren die Art des Appellcharakters eines Wortes zunehmend. In vielen Fällen läßt sich an den Glossen usw. der Prozeß ablesen, in dem appellative Aspekte wie Parteilichkeit, Stigmatisierung als Euphemismus oder als polemisch u.a. vom (explizit kritischen) Kontext auf die Lexikoneinheit übergehen; und zwar in einem Maße, daß Lexikologen und Lexikographen hinfort mit diesen appellativen Aspekten als realen Dimensionen der Wortbedeutung umgehen müssen.

Interessengebundenheit und Wertungspotentiale von Wörtern werden zu einem großen Teil in der Diskussion in den Massenmedien geschaffen. Es stellt sich die Frage: wie bzw. wie noch? Wenn sprachkritische Kommentare und Glossen ein relativ einheitliches Schema von Positiv-negativ-Bewertungen, von "richtigen" und "falschen" Wörtern propagieren - welche anderen, vielleicht sogar konträren Bewertungsschemata werden über andere Textsorten in die Diskussion eingebracht?

\section{Bezeichnungsvarianten in Expertentexten}

4.1 Die vielleicht effektivste weil, sanktionsfähige Textsorte mit der meisten Durchsetzungsmacht in bezug auf die Festlegung der Bewertung von Ausdrücken sind amtliche Texte wie Gesetze und Verordnungen. Die Freiheiten, solche Texte paraphrasiert mit anderen, selbst gewählten Ausdrücken wiederzugeben, sind - dies betrifft vor allem Journalisten - äußerst beschränkt; es besteht ein gewisser Zwang zum wörtlichen Zitat. Die Bevorzugung bzw. ausschließliche 
Verwendung z.B. des Wortbildungselements Kern- im Zusammenhang von Kernenergie und Kernkraftwerk anstelle von Atom- ${ }^{11}$ und von Abfall- vor allem als Simplex anstelle von Müll in amtlichen Texten führt zur Fixierung des Appellcharakters als an 'sachliche, korrekte, exakte, emotional neutrale und objektive' Argumentation gebunden.

4.2 Die zweite durchsetzungsmächtige Textsorte sind Darstellungen, Gutachten, Stellungnahmen usw. von Wissenschaftlern und Technikexperten aus der Industrie. Sie treten mit ihren Entscheidungen für oder gegen bestimmte Konkurrenzwörter in der Öffentlichkeit selten direkt in Erscheinung. Sie liefern aber für alle, auch für ihre eigenen Kritiker aus den Reihen der Umweltschutzverbände, nahezu das gesamte Quellenmaterial. ${ }^{12}$ Es läßt sich nachweisen, daß auch in den kritischsten Büchern, Broschüren und Lexika aus der Feder von Umweltschützern die Konkurrenzwörter mit gleicher Charakterisierung und Appellativität verwendet werden wie von den Fachexperten in den Ausgangstexten, weil die kritischen Texte sich innerhalb einer Popularisierungsskala auf die kritisierten Texte beziehen (müssen).

Die auf diese Weise in die breite Öffentlichkeit weitergereichte Charakterisierung bestimmter Ausdrücke läßt sich angeben als 'wissenschaftlich begründet, unangreifbar, sachlich objektiv und emotional neutral' oder, um die Appellativität zu paraphrasieren: "Willst du, daß man dich als ernsthaften, informierten und nicht polemischen Diskussionspartner ernst nimmt, dann sprich von Kernenergie, nuklearer Entsorgung und Problemabfall anstatt von Atomkraft, Atommüll oder Giftmüll."

Ein Beispiel ist die Bezeichnung Kernbrennstoffkreislauf. Alternative Bezeichnungen hierzu werden in kritischen Publikationen sporadisch ganannt: Brennstoffspirale oder Plutoniumfabrikation, sie werden aber nicht konsequent anstelle von Brennstoffkreislauf verwendet. Das liegt offenbar daran, daß auch von den Kritikern die Reservierung von Wissenschaftlichkeit, Objektivität, Neutralität für eine der Bezeichnungsvarianten nicht in Frage gestellt wird. Dieser Variante gegenüber müssen alle anderen als zu bildhaft, emotional, polemisch und einseitig erscheinen. Auf wissenschaftliches Prestige und

11 'Atomgesetz' und 'Atomrecht' sind historisch begründete Ausnahmen, dazu s. Abschnitt 5.4 .

12

Vgl. die zu vielen einzelnen Lexikon-Artikeln angegebenen Literaturnachweise in Schwann, Taschenlexikon Umweltschutz und Umwelt-Lexikon, hrsg. von der Katalyse-Umweltgruppe (s. Quellenverzeichnis). 
den Eindruck der Sachlichkeit in der Argumentation wollen und können die Kritiker aber nicht verzichten; deshalb verwenden sie auch solche Bezeichnungen, die ihrer Ansicht nach eigentlich verschleiern oder eine unangemessene Sehweise eines Sachverhalts erzeugen.

4.3 $\mathrm{Zu}$ allem Unglück ergeben sich zwischen diesen beiden mächtigsten Textsorten nicht selten Zusammenhänge der Art, daß amtliche Texte Formulierungen aus Expertentexten aufgreifen und sich zu eigen machen. Obwohl es eigentlich auf der Hand liegt, fällt dies nur ausnahmsweise auf, wie einem Kommentator (Martin Urban) in der Süddt. Zeitung vom 27.11.1987 (S. 4) anläßlich der Nordsee-Konferenz in London:

Manche Regierung macht sich dabei zum Lautsprecher ihrer Industrieverbände. Besonders unverfroren gibt sich die Confederation of British Industry, CBI. Deren Schlagworte finden sich, wie in der Fachzeitschrift "Wattenmeer international" (2, 1987) soeben dokumentiert wurde, bereits im offiziellen Bericht zur Nordseekonferenz, einem Kompromißpapier aller Anrainer. Dort wird, entsprechend der britischen Vorstellung, von der "legitimen industriellen Nutzung", sprich: Verschmutzung der Nordsee, gesprochen.

Eine weitgehende Übereinstimmung in der Wortwahl zwischen Experten und Gesetzgebern läßt sich an umweltrechtlichen Verordnungen auch innerhalb der Bundesrepublik feststellen.

Auffallen kann die Fixierung von bestimmten Sehweisen, appellativen und evaluativen Funktionen mittels geeigneter Bezeichnungen und damit die Interessengebundenheit von Wortverwendungen eigentlich nur solchen Sprechern, deren individueller Wortschatz alternative Bezeichnungen für denselben Sachverhalt enthält. Umweltwortschatz wird nicht nur in den zwei zuletzt behandelten Textsorten vermittelt und dort als Menge von Ausdrucksmitteln mit relativ großer Verbindlichkeit und eher geringerer Variabilität präsentiert. Einen nicht geringen Anteil an der Wortschatzvermittlung und damit an der Durchsetzung bestimmter Ausdrücke haben die seit etwa 15 Jahren existierenden Umweltlexika, -nachschlagewerke und glossare, denen ich mich nun zuwenden möchte. 


\section{Bezeichnungsvarianten in Umweltlexika}

Wörterbücher und Lexika bilden im Textsortenspektrum der Umweltdiskussion eine Klasse, die für die verständliche Vermittlung von Wissen, und zwar von staatsbürgerlich äußerst relevantem Wissen, zentrale Bedeutung hat. Den zehn zwischen 1973 und 1987 erschienenen und von mir untersuchten Lexika ${ }^{13}$ ist gemeinsam, daß sie sich an "Leser anspruchsvollerer Zeitschriften- und Zeitungsartikel", an "Lehrer, Studenten, Schüler, Praktiker in Planungsgremien und Behörden", an "interessierte Laien, Techniker, Politiker" - eigentlich also an jeden wenden.

Der didaktische Aspekt und die Unterrichtseignung wird in einigen der Lexika besonders betont. Darüber hinaus sind ihnen formale Eigenschaften gemeinsam, nämlich die alphabetische Ordnung nach Stichwörtern.

Zwei der zehn Lexika sind nicht im Buchhandel erhältlich, sondern werden bzw. wurden auf Anfrage vom Bundesumweltministerium, früher vom Bundesinnenministerium, und vom Kernforschungszentrum Karlsruhe verschickt. So weit erkennbar, waren nur in einem Fall (Meyers Kleines Lexikon Ökologie) professionelle Wörterbuchmacher beteiligt, was sich in der stärkeren Einbeziehung sprachlicher Aspekte (Grammatik, Aussprache, auch Angabe von Synonymierelationen) bemerkbar macht. Bei den Autoren aller übrigen Lexika dürfte es sich um Fachleute handeln, bei zweien mit zusätzlicher pädagogischen Erfahrung (rororo) und in Umweltschutzgruppen aktiv (KiWi).

\subsection{Stichwörter, Verweise und sog. Synonyme}

Umweltlexika vermitteln erwartungsgemäß überwiegend Sachinformationen, und Lexikonautoren formulieren erwartungsgemäß so objektiv und wertneutral wie möglich. So wird man bis auf wenige Ausnahmen (rororo) keine deutlichen parteiischen Aussagen und Urteile über Sach- und Problemverhalte finden. Es fragt sich, ob es hinsichtlich der Strittigkeit vieler "Sach"-Verhalte im Umweltbereich eine wirklich unparteiische Darstellung überhaupt geben kann und ob

13 S. Quellenverzeichnis; inzwischen ist noch ein weiteres erschienen: H. Walletschek / J. Graw (Hg.): Oko-Lexikon. Stichworte und Zusammenhänge. München (Beck'sche Reihe 344) 1988. 
sich Parteilichkeit, bestimmte Sehweisen und Werturteile nicht auch in den scheinbar unverdächtigen Entscheidungen finden, die Lexikonautoren zu treffen haben, z.B. in der Auswahl der Stichwörter.

Ein Lexikonartikel ist, wie ich ihn auffasse, ein Text zu einem Thema, das durch das Stichwort angegeben wird. Der Text darf dieses Thema nicht verfehlen und er muß möglichst kurz sein, d.h. er muß das Wichtigste zum Thema angeben. Was in Lexikonartikeln weggelassen ist, hält ein Leser mit der üblichen Erwartung gegenüber dieser Textsorte für das Unwichtige. Weitergehende Vorgaben, wie es sie in der Sprachlexikographie gibt, sind hier nicht anzunehmen. Das muß zwangsmäßig dazu führen, daß bei Themen bzw. Stichwörtern, die strittig sind und bei denen mehr als eine Sehweise möglich ist, ganz unterschiedliche Texte verfaßt werden, je nachdem welche Sehweise des Themas der Autor vermitteln möchte. Die Tendenz zur zusammenfassenden Behandlung größerer Sachgebiete ist in den untersuchten Lexika unterschiedlich stark und wird mit Hilfe von Unterstichwörtern, die dem alphabetischen Prinzip nicht folgen müssen, umgesetzt. Solche Unterstichwörter habe ich im Folgenden mitberücksichtigt, weil die Sehweise eines Themas wesentlich auch durch die Art der Strukturierung des Erläuterungstextes bestimmt wird.

Unterstichwörter sind nicht durch Fettdruck hervorgehoben und stehen auch nicht in der alphabetischen Reihe, sondern werden im Rahmen eines Sachzusammenhangs angeführt und dort auch mit einem eigenen Erläuterungstext versehen. Dieses Verfahren wird nur von zwei der Lexika (Meyers und rororo) überhaupt genutzt. Als Unterstichwort wird einer Bezeichnung eine gewisse Thema-Würdigkeit zugesprochen und zugleich wird ein zunächst assoziatives, vielleicht sogar potentiell semantisches Netz aufgebaut, das die Sehweise des Sachzusammenhangs prägt.

Ein Beispiel: Das Hauptstichwort Müll wird bei rororo recht ausführlich erläutert; am Schluß des Textes folgen zwei Abschnitte mit den Unterstichwörtern Müllvermeidung und Müllwiederverwertung, gewissermaßen als den zwei Dingen, die man mit Müll tun sollte. Das Beispiel macht deutlich, wie assoziative Verknüpfungen mehrerer Ausdrücke deren Appellfunktion herausstellen und verstärken. Müll erhält durch die zwei Unterstichwörter eine neue und noch ungewohnte deontische ${ }^{14}$ Bedeutungskomponente. Konstruiert man als Gegenbeispiel den Fall, daß in einem Lexikon zum Hauptstich-

Deontische Wörter hat Hermanns 1986 beschrieben. 
wort Müll die Unterstichwörter Müllabfuhr, Mülleimer, Müllverbrennung, und zwar nur diese, aufgeführt werden, legt dies als deontische Bedeutung von Müll nahe: 'Müll ist etwas, was man loswerden muß und das dann auch tatsächlich verschwindet'.

Die Anlage solcher Netze ist offensichtlich interessengebunden. Ich wüßte gar nicht, wie ein Lexikon-Autor dabei 'objektiv' sein könnte; trotzdem halte ich sach- oder besser problemorientierte (s. unten) Verknüpfungen von Stichwörtern für wünschenswert, denn: Welche Interessen verfolgt oder unterstützt das entgegengesetzte Verfahren z.B. des Herder Lexikons, das den thematischen Zusammenhang Umwelt in "1800 Stichwörter" atomisiert, d.h. in isolierte Einzelbegriffe wie Müll, Müllabwurfanlage, Müllzerkleinerer, Müllschlucker u.a., von denen nicht einmal ein Verweispfeil einen $\mathrm{Zu}-$ sammenhang mit Abfallverwertung oder Recycling andeutet? Im übrigen kommt diese Stichwortreihe dem oben konstruierten Gegenbeispiel (Müllabfuhr, Mülleimer usw.) recht nahe, denn sie schränkt die Sehweise bei Müll auf das 'Weg damit' ein.

Einen Ausdruck als bloßes Verweisstichwort aufzuführen, interpretiere ich als relative Abwertung dergestalt, daß dieser Ausdruck als nicht thema-würdig behandelt wird. Dieser Ausdruck steht zwar fettgedruckt in der alphabetischen Reihe, er hat aber keine eigene Erläuterung, sondern er verweist den Leser auf ein oder mehrere andere Hauptstichwörter. Die Verweise beziehen sich z.T. auf Wörter innerhalb des hier betrachteten Wortfelds, z.B. von Giftmüll auf Sonderabfälle (KiWi), z.T. auch auf außerhalb liegende Ausdrücke, z.B. auf Entsorgung oder Recycling. Diese Relationen und was sie an Assoziationslenkung implizieren, konnte ich in diesem Rahmen nicht mehr dokumentieren.

Außerdem gibt es Bezeichnungen, die nicht zu dem vom Lexikon bevorzugten Erklärungsstil (vgl. unten) passen, so gilt z.B. Giftmüll offensichtlich als unpassendes Stichwort für einen Erklärungstext, der sich sachlich und stilistisch eng am Abfallbeseitigungsgesetz orientiert.

Atommüll ist die Bezeichnung mit den meisten Verweisen, d.h. ihre Existenz in der öffentlichen Diskussion ist zwar nicht zu leugnen, als angemessene Themenbezeichnung soll aber radioaktiver $A b-$ fall gelten. Nur die Lexika von Innenministerium und Kernforschungszentrum verweigern Atommüll jegliche Beachtung und stigmatisieren diese Variante somit für ihre eigene Sprechergruppe. 
Trotz der bei Lexika vorausgesetzten Neutralität finden sich in einzelnen Fällen und ohne erkennbare Systematik Äußerungen, die sich auf den Wortgebrauch beziehen. Diese Ausdrücke werden in den Lexika durchweg als Synonyme bezeichnet. Sie werden kursiv oder eingeklammert meist direkt hinter das Hauptstichwort gesetzt, of $t$ ganz ohne Kommentierung, aber auch mit mehr oder weniger deutlich bewertenden Zusätzen. Die Kommentare beziehen sich auf Kriterien wie "offiziell", "korrekt", "genau/ungenau", "passend" und belegen insgesamt mit ihrer Widersprüchlichkeit die Brisanz ganz bestimmter Varianten: es sind dies tatsächlich die Paare Abfall/Müll und Atom-/Kern-. Es lohnt sich, die Art der Kommentare näher zu betrachten (s. 5.3.).

Mit der Besetzung dieser vier Kategorien (Stichwort, Unterstichwort, Verweisstichwort, abgewertetes Synonym) durch bestimmte, z.T. umstrittene Bezeichnungen wird ein Sach- bzw. Problembereich konzeptualisiert. Die gewählten Stichwörter modellieren sein Profil und das Feld aller Stichwörter eines thematischen Bereichs repräsentiert ihn dem informationssuchenden Leser gegenüber.

\subsection{Stichwortfelder}

Ich habe in den zehn Umweltlexika die Behandlung zweier Wortfelder untersucht, und zwar solche, in denen auffallend viele Bezeichnungsvarianten für denselben Sachverhalt existieren, wenn man vom Sprachgebrauch der Massenmedien ausgeht.In einem Anhang sind beide Wortfelder tabellarisch dargestellt. Zum einen sind dies die Wörter Abfall und Müll als Simplizia und als Basiswörter der folgenden Zusammensetzungen:

Chemieabfall/-müll, Giftabfall/-müll, Gewerbeabfall/-müll, Industrieabfall/-müll, Problemabfall/-müll, Sonderabfall/-müll, Hausmüll und Sperrmüll.

Außerdem habe ich Anzahl und Art der als Stichwörter verzeichneten rechtsläufigen Ableitungen, etwa Abfallbeseitigung oder Giftmüllskandal, berücksichtigt, weil auch mit ihnen die Konzeptualisierung des Problemverhalts in entscheidender Weise gesteuert wird.

Das zweite untersuchte Wortfeld gruppierte sich um die Varianten Atomkraft und Kernkraft, Atomenergie und Kernenergie. Auch hierbei ging es mir um das Feld der Stichwörter, die mit den Elementen Atom-, Kern- und Nuklear- als Brstimmungswörtern eine Art Text 
im Text bilden, an dem sich die unterschiedliche Bewertung der Varianten ablesen läßt. Gewissermaßen auf der Grenze zwischen beiden Wortfeldern befindet sich Atommüll gegenüber radioaktiver Abfall; sie sind in jede der beiden Tabellen (s. Anhang) aufgenommen.

Meine Untersuchung konzentriert sich auf die Perspektiven, die mittles der Auswahl von Stichwörtern, Unterstichwörtern, Verweisen und Kommentaren zu Synonymen auf einen Sach- und Problembereich hin angelegt werden.

Bei sieben der zehn Lexika besteht der Anspruch, den gesamten Sachverhaltsbereich des Themas Umwelt abzudecken, zumindest werden keine Einschränkungen oder absichtlichen Auslassungen angegeben. Das Lexikon des Kernforschungszentrums Karlsruhe bezieht sich nur auf das Thema Kernenergie, enthält also nichts zum Thema Müll, aber auch nichts zu den Stichwörtern radioaktiver Abfall bzw. Atommüll. Das ist, gemessen am stillschweigend (d.h. durch die Wahl der Textsorte Lexikon) bekräftigten Anspruch, umfassend zu informieren, eine bemerkenswerte Auslassung.

Das Bundesministerium des Innern gab eine kleine Reihe von Taschenbüchern mit dem Titel "Was Sie schon immer über XY wissen wollten" heraus, in der jeder Band einem Teilthema, z.B. "Luftreinhaltung", "Auto und Umwelt" oder "Wasser und Umwelt" gewidmet ist. Davon habe ich zwei ausgewählt, einmal das allgemeinste "Was Sie schon immer über Umweltschutz wissen wollten" und zum anderen das speziellere "Was Sie schon immer über Abfall und Umwelt wissen wollten".

In den Tabellen sind beide in einer Rubrik zusammengefaßt. Interessant ist, daß in beiden der Kernenergie-Komplex total ausgeblendet wird und offensichtlich existiert auch kein eigenes Bändchen zu diesem Thema. Über Kernenergie haben wir Bürger anscheinend immer schon nichts wissen wollen. Das Umweltministerium hat die Lexikonreihe vom Innenministerium übernommen. Eines der untersuchten Lexika (ABC-Umweltschutz) fällt als DDRPublikation aus der bundesdeutschen Diskussion heraus; zum Vergleich ist es aber interessant, denn auch hier wird das KernenergieThema völlig ignoriert. Schon beim Weglassen beginnt - zumindest im Lexikon - der interessenabhängige Umgang mit Wörtern. 


\subsection{Müll oder Abfall?}

Im ältesten der zehn Lexika (Herder) wird Müll noch ganz ohne Anzeichen von Strittigkeit als

org. u. anorg. Abfall (unterschiedlicher Zusammensetzung) ... der Haushaltungen, Gewerbe, Betriebe u. von der Straße (S. 138)

paraphrasiert; auch das zweitälteste (Schwann) erklärt Müll als

Abfall aller Art, der in seiner Zusammensetzung sehr unterschiedlich ist ... (S. 150)

Nach 1980 aber wird Strittigkeit und damit Interessengebundenheit explizit. Bei rororo heißt es:

Die offizielle Bezeichnung 'Sonderabfall' klingt eigentlich viel zu harmlos, wenn man die katastrophalen Folgen für Umwelt und Gesundheit bedenkt, die von den jährlich in der Bundesrepublik anfallenden $150 \mathrm{Mio} . t$ Industriemüll ausgehen. Für mindestens 5 Mio. $t$ davon ist wohl nur der Ausdruck 'Giftmüll' passend. (S. 113)

Die Kommentare aus dem Lexikon des Innenministeriums hören sich fast wie eine Antwort hierauf an, obwohl Sonderabfall als eigenes Stichwort geführt wird, heißt es unter dem Stichwort Abfall-Arten:

Der Begriff "Sonderabfälle" wird im Gesetz genausowenig verwendet, wie die Begriffe "Müll" oder "Hausmüll". (S. 48)

Anschließend werden verschiedene Klassifikationsmöglichkeiten für Abfallarten genannt, wie sie in Abfalltechnik und Kommunalverwaltung üblich sind, d.h. dem Leser wird als Alternative zu Sonderabfall und Müll eine Fachterminologie präsentiert, die in der breiten Mediendiskussion keine Rolle spielt, oder wenn, dann in einer Glosse mit der Überschrift "Berge von Sprachmüll" (Süddt. Ztg. 18.1.1984).

Während rororo für Giftmüll als den "passenden" Ausdruck plädiert und seine Stichwortwürdigkeit gegenüber Sonderabfall rechtfertigt, wird Giftmüll beim Innenministerium nur als Stichwort Giftmüllskandale erwähnt. Grammatisch nicht korrekt beginnt der Erläuterungstext wie folgt;

Bezeichnet in der Regel die ungeordnete und gesetzwidrige Beseitigung industrieller Sonderabfälle: (im Zuge des gerade beginnenden Vollzugs des 1972 verabschiedeten Abfall-Beseitigungsgesetzes wurden jedoch auch Praktiken mit diesem Begriff belegt, die rechtlich einwandfrei wa- 
ren, auf dem Hintergrund einer zunehmenden Sensibilisierung der Öffentlichkeit aber als ökologisch nicht mehr verantwortbar angesehen wurden ..

weiter unten:

Die G. und die Berichterstattung haben sehr viel zur Ausbildung eines Umweltbewußtseins in der breiten Öffentlichkeit und zur Verbesserung von gesetzlichen und administrativen Maßnahmen bei der Abfallbehandlung beigetragen. (S. 118)

und an anderer Stelle wird Müll-Lawine erläutert als

Volkstümliche Kennzeichnung der Entwicklung des AbfallAufkommens. (s. 156)

Was zeigen die gegensätzlichen Kommentare dieser beiden Lexika? Die rororo-Autoren verteilen Prädikate: "eigentlich viel zu harmlos" und "passend" und stellen dem allgemeinen, breiten und konsentischen Sprachgebrauch des "wir" ("was wir Müll nennen") den besonderen, eingeschränkt gültigen und in gewisser Weise abweichenden Sprachgebrauch der "Behörden und Fachleute" gegenüber ("heißt im Sprachgebrauch der Behörden und Fachleute Abfall"). Das heißt, sie kämpfen für die von ihnen bevorzugten Bezeichnungen, indem diese als die sowieso üblicheren markiert werden, deren Gebrauch sich die Leser deshalb leicht anschließen können sollen.

Der Kampf der ministeriellen Autoren ist subtiler. Sieht man von dem ambivalenten "volkstümlich" ab (ist dies ein aufwertendes oder ein abwertendes Prädikat?), verzichten sie auf explizite Prädikate. Statt dessen entwickeln sie in ihrer Erklärung zwei unterschiedliche Sehweisen des Themas Giftmüll bzw. Beseitigung industrieller Sonderabfälle. Die eine Sehweise und ihre Vermittlungsformen in der öffentlichen Diskussion werden explizit formuliert mit den Ausdrücken: "Skandal", "auf dem Hintergrund", "zunehmende Sensibilisierung der Öffentlichkeit". Damit wird die Sehweise mitsamt der sie repräsentierenden Bezeichnung Giftmüll distanzierend als Möglichkeit charakterisiert, praktisch aber, quasi als 'reine Ansichtssache', abgewertet.

Die andere Sehweise wird implizit vertreten mittels der Ausdrücke: "gesetzwidrig", "Vollzug des verabschiedeten Gesetzes", "rechtlich einwandfrei", "gesetzliche und administrative Maßnahmen bei der Abfallbehandlung" und "Abfall-Aufkommen". Es handelt sich um die administrative und sanktionsfähige Sehweise mit den Vermittlungsformen 'Maßnahmen' und 'Gesetz', die dem ganzen Lexikon 
zugrunde liegt und der der Leser sich nicht entziehen kann. Bedenkt man wiederum, wieviel Autorität und Prestige lexikonartige Texte haben, wird klar, daß gerade die implizit durchgängigen Sehweisen nicht als Meinungen, sondern als Wahrheiten aufgefaßt werden und mit den Sehweisen auch die sie repräsentierenden Bezeichnungen als der wahre und autoritativ abgesegnete Sprachgebrauch.

\subsection{Atomkraft oder Kernenergie? (mit einem historischen Exkurs)}

Ich möchte dem Müll-Beispiel noch ein Beispiel aus dem anderen Themenbereich an die Seite stellen und fragen: Wie sieht es mit der Charakterisierung der Varianten Atomkraft, Atomenergie vs. Kernkraft, Kernenergie aus?

Das Herder-Lexikon entscheidet sich überall für Zusammensetzungen mit Kern- als Stichwort und setzt regelmäßig die unkommentierten "Synonym"-Angaben mit Atom- dahinter. Desgleichen Schwann: hinter dem Stichwort Kernkraftwerk (S. 116) steht Abkürzung $K K W$, auch Atomkraftwerk; Schwann scheint übrigens vieles schematisch von Herder abgeschrieben zu haben. Ebenso wie diese beiden verfährt das Lexikon von Schneider: für Kern- und gegen Atom-. rororo dreht das Verhältnis von aufwertendem Stichwort und abwertendem Synonym um, schreibt zum Stichwort Atomkraftwerk: Atomkraftwerke (auch Kernkraftwerke genannt) (S. 39) und Atomenergie (auch Kernenergie genannt) (S. 39), kommentiert diese Entscheidung aber ebenfalls nicht.

Daß das Kernforschungszentrum dem Element Kern- den absoluten Vorrang einräumt, verwundert nicht, jedoch gibt es in kerntechnischen Anlagen keine Unfälle, sondern nur nukleare Ereignisse (S. 93f.). Es bringt mit nuklear also noch eine dritte Variante ins Spiel, die sonst nur spezielle und im allgemeinen unstrittige Forschungsdisziplinen, etwa Nuklearmedizin, charakterisiert.

Die Tabelle zeigt, daß nuklear (als Adjektiv und als Wortbildungselement) in den Lexika noch relativ wenig Aufmerksamkeit geschenkt wird. Dort ist nuklear gegenüber Atom-, atomar und Kernals besonders fachsprachlich charakterisiert. Die Belege des Mannheimer Korpus ${ }^{15}$ zeigen dagegen, daß nuklear ganz überwiegend dem

15 Dort sind Texte der deutschen Gegenwartssprache von 1945 bis heute in verschiedenen Textkorpora maschinell gespeichert. S. LDV-Info 1, 2 und 4, hrsg.. vom IDS $1981 \mathrm{ff}$. 
militärischen Bereich zugeordnet wird. Erst in allerjüngster Zeit wird nuklear daneben auch zur Kennzeichnung des Zusammenhangs mit dem Energiethema verwendet.

Die Textbelege lassen vermuten, daß nuklear noch als appellativ neutral gilt, als fach- und damit sachbezogen und noch nicht vom Parteilichkeitszwang semantischer Kämpfe affiziert. Dies wird sich natürlich rasch ändern, wenn nuklear von bestimmten Sprechergruppen bevorzugt und in parteiischer Weise verwendet wird.

Im Bereich des Umweltwortschatzes ist generell $\mathrm{zu}$ beobachten, daß die Fachsprachlichkeit eines Ausdrucks, d.h. die ihm unterstellte Zugehörigkeit $\mathrm{zu}$ einem sachbezogenen, wertneutralen und unpolitischen Bereich der Kommunikation, zur Euphemisierung brisanter Sachverhalte benutzt werden kann.

Das Lexikon der Katalyse-Umweltgruppe schlägt sich durchgehend auf die Seite der Kern-Verwender und kommentiert die Bezeichnung Atomkraftwerk als

Umgangssprachliche Bezeichnung für Kernkraft, die physikalisch nicht korrekt ist. (S. 38)

Meyers Autoren scheinen dies zu übernehmen; dort steht unter dem Stichwort Kernenergie: ungenaues Synonym: Atomenergie (S. 135).

Was hier mit "ungenau" und "physikalisch nicht korrekt" gemeint ist, erkläre ich folgendermaßen: Die Motiviertheit der Zusammensetzung, d.h. das, was der Nicht-Linguist als die innere Logik eines zusammengesetzten Wortes auffaßt, wird durch die Bildung seiner Paraphrase überprüft, etwa: 'Atomenergie ist die Energie, die aus Atomen erzeugt wird' und 'Kernenergie ist die Energie, die aus Kernen, Atomkernen natürlich, erzeugt wird'. Danach wäre von den Paraphrasen eine falsch, soweit erkennt das auch der gebildete Nicht-Fachmensch; Atome bestehen aus mehr als nur einem Kern und die Energie wird irgendwie durch Spaltung dieses Kerns, nicht des Atoms als Ganzem, erzeugt.

Linguistisch ist dazu zu sagen, daß zusammengesetzte Wörter selten die Kurzform eines definierenden Satzes sind und auch nicht dessen Resultat, sondern ihre Form häufig in historischen Prozessen entwickelt wird. Daß irgendetwas Geschichtliches an der Form von Zusammensetzungen wie an Wörtern überhaupt beteiligt sein könnte, ist den meisten Sprachteilhabern nicht bewußt, sie suchen die Motivierung ausschließlich auf der synchronen Ebene. 
Bei Atomenergie und Kernenergie hat allerdings die Vorgeschichte beträchtlichen Einfluß auf die heutige Verwendung und appellative Charakteristik.

\section{Historischer Exkurs}

Beide Varianten sind formal gesehen Verkürzungen der früheren Bezeichnung Atomkernenergie; Atomenergie gehört in ein Wortbildungsparadigma, das in den 50er/60er Jahren äußerst produktiv und gegenüber Kern- dominant war: Atomgesetz (1959), Atomminister (Ministerium für Atomfragen), Atommeiler, Die Atomwirtschaft (so hieß eine damalige Fachzeitschrift). Eine offene Reihe mit Atombildeten auch umganssprachliche Ausdrücke, die offenbar den gewaltigen Eindruck der Atombombe metaphorisch verarbeiteten, sei es zur Charakterisierung von Fußballspielern (Atomkanonier), des Wetters, harten Brots, einer neuen Rosensorte (Atombombe) (wie ein "mächtiger schalachroter Glutball" - Die Zeit 19.9.1957, 4) oder weibliche Körperteile aus Männersicht. ${ }^{16}$ Atom- funktionierte in der Umgangssprache als steigerndes, aber in der Wertung ambivalentes Präfix. ${ }^{17}$

Dem gegenüber wurde die öffentlich-politische Diskussion in den $50 \mathrm{er} / 60 \mathrm{er}$ Jahren beherrscht von den Verhandlungen um den Atomwaffensperrvertrag, die noch unter dem Schock von Hiroshima und Nagasaki stattfanden und damit von der Ambivalenz "des Atoms": Segen oder Fluch für die Menschheit, Energie und Wohlstand oder Bombe und Tod. ${ }^{18}$ Diese, als schicksalhaft empfundene Zweischneidigkeit liegt auch in Formel von der "friedlichen Nutzung der Atomenergie (heute: Kernenergie)" zugrunde.

Nach meinen bisherigen Quellenstudien war die oft vermutete "Atomeuphorie" dieser Zeit nur eine Seite des allgemeinen gesellschaftlichen Bewußtseins, und zwar möglicherweise die politisch verordnete; die andere Séite bildete der Schock durch die Atombombe.

Alle Beispiele: Küpper 1982, Bd. 1, S. 194 / 195.

17

Ich stütze mich hier, außer auf das Mannheimer Korpus, auf Stichproben aus: Die Zeit, Januar 1954 bis Februar 1963, Der Spiegel 1968. Eine systematischere Auswertung auch anderer Zeitungen war im Rahmen dieser Arbeit nicht möglich.

Vgl. die Titel, die Kindlers Literaturgeschichte der Gegenwart, Bd. 11, S. 118 - 121 und S. 158 - 162 als naturwissenschaftliche bzw. technische Sachbücher der Bereiche "Physik" und "Atomkraft und Weltraumfahrt" anführt. 
Diese Schattenseite stand der fortschreitenden Propagierung der Atomenergie mehr und mehr entgegen, denn die Bezeichnung Atomwurde die Assoziation an die Bombe nie ganz los.

Zeitlich etwa parallel zu den Atom-Zusammensetzungen, aber abseits der öffentlichen Diskussion bildete sich auch Kernenergie aus Atoménergie, und zwar vorwiegend in der Fachkommunikation, wo es noch eine ganze Reihe anderer Kern-Zusammensetzungen gibt, bei denen die regelmäßige Übersetzung von engl. nuclear durch Kerneine wichtige Rolle spielt. Gerade in der "Eindeutschung" angelsächsischer Fachausdrücke sah man ein wesentliches Instrument, um die Akzeptanz der Atompolitik zu fördern. ${ }^{19}$

Etwa um 1970 ändert sich das Häufigkeitsverhältnis von Atomund Kern- zugunsten des letzteren. In den ältesten Teilkorpora des Mannheimer Korpus (Texte ab 1945) ist Atomenergie viermal häufiger als Kernenergie; seit den 80er Jahren ist aber Kernenergie fünfbis sechsmal häufiger als Atomenergie. Ganz ähnlich sieht es bei Atomkraft und Kernkraft aus; letzteres kommt insgesamt 296mal vor, Atomkraft nur $159 \mathrm{mal}^{20}$

Das deutsche Atomforum (ein Zusammenschluß von Vertretern aus Industrie, Wirtschaft, Verwaltung und Politik) geht ab 1970/72 in ihren für die Öffentlichkeit bestimmten Schriften und in Presseverlautbarungen dazu über, Ausdrücke mit Kern- anstelle von Ausdrücken mit Atom- vorzuziehen. So lautet der Titel einer von ihm 1971 herausgegebenen Broschüre: "Kernfragen. Antworten auf Fragen zur Kernenergie" (Röthlein 1979, 68ff.). Die Angst vor Radioaktivität und Atom-Katastrophen hielt man für die verlängerte Angst vor der Atombombe und hoffte, mit dem Bezeichnungswechsel mindestens auch, den industriell-kommerziellen Einsatz der Atomenergie aus dieser gedanklichen und gefühlsmäßigen Verwandtschaft herauszulösen (so Röthlein 1979, 68).

Die ursprünglich fachsprachliche Bezeichnung spielt in der öffentlichen Diskussion mittlerweile die größere Rolle - auch ein Indiz für den größeren Einfluß, den die Industrie und jedes Image von Wissenschaftlichkeit in der Diskussion ausübt. Die Kern-Zusammensetzungen gelten jetzt, das zeigen die Bewertungen in den Lexika, als

19 Vgl. Höcker 1957; Ischreyt 1958 und Lötje 1977.

20 Die Zahlen müssen auf dem Hintergrund der Tatsache gesehen werden, daß das Mannheimer Korpus für die 80er Jahre mehr Zeitungstexte enthält als für die frühen 70er Jahre. 
die seriösen, neutralen, objektiven, korrekten, genauen Bewertungen, die sich auch auf die Sprecher übertragen, die Kern- verwenden. Dem gegenüber wird der Gebrauch von Atomkraft schon fast mit der Äußerung des Slogans "Atomkraft - nein danke!" gleichgesetzt. Es wird einerseits zum Fahnenwort, an dem sich die Gegner dieser Energieart und alle Öko-, Friedens-, Alternativ-Bewegten erkennen sollen, z.B. wenn die SPD oder Volker Hauff nach Tschernobyl plötzlich den Ausstieg aus der Atomenergie bejahen. Es signalisiert andererseits, die, die es verwenden, seien polemisch, unsachlich, emotional, kurz: in dem, was sie äußern, nicht ernst zu nehmen.

\subsection{Sachorientierte und problemorientierte Darstellung}

Bis hierher habe ich mich hauptsächlich mit den Konzeptualisierungen, Fokussierungen und Akzentuierungen beschäftigt, die in den Umwelt-Lexika durch die Auswahl der Stichwörter, ihre Zusammenstellung zu Feldern und durch die Abgrenzung gegenüber abgewerteten "Synonymen" bewirkt werden. Man kann aber über interessengebundene Sprachlenkung in Lexika nicht reden, ohne auch auf die Art der Darstellung in den Lexikonartikeln einzugehen.

In den Lexika sind deutlich zwei Seh- und damit Darstellungsweisen zu unterscheiden. Die eine nenne ich zusammenfassend sachorientiert. Die Sache kann ein technischer Zusammenhang sein, z.B. das Funktionieren eines Kernkraftwerks oder die einzelnen Schritte, die beim Recycling zusammenwirken müssen. Die Sache kann aber auch ein administrativer Zusammenhang sein, etwa die Handlungen, die das Abfallbeseitigungsgesetz vorschreibt oder die Klassifikationen von Gegenständen, z.B. Abfällen, nicht so wie die Alltagserfahrung oder die öffentliche Diskussion sie vornimmt, sondern so, wie sie eine Verordnung erstellt.

Sachorientierte Darstellung von Themen, die gesellschaftlich umstritten oder problemgeladen sind, ist zugleich eine idealtypische Darstellung, bei der soweit wie möglich von strittigen und problematisierenden Beurteilungen abstrahiert wird; ihr liegt die Annahme zugrunde, man könnte eine Sache (ein Kraftwerk, ein Entsorgungskonzept, ein Biotop) losgelöst von allen sie betreffenden Interessen, Belangen, Begründungen und Schlußfolgerungen betrachten.

Ihr gegenüber steht die problemorientierte Darstellung. Problemorientierung im Lexikon liegt dann vor, wenn die Sehweise der Öf- 
fentlichkeit, der Betroffenen oder der Adressaten (d.h. die Sehweise der Leser des Lexikons) anstelle der Sehweise von Fachleuten und Verwaltungsexperten die Erläuterung eines Stichworts prägt. Nicht die Sache an und für sich wird betrachtet, sondern die Sache im $\mathrm{Zu}$ sammenhang einer mehr oder weniger kontroversen Diskussion einschließlich der Konsequenzen, die etwa Recycling für den Alltag des Einzelnen haben kann.

Beide Darstellungsformen kommen in den Umweltlexika gemischt vor, wie bei diesem Beispiel aus Herder:

Industriemüll, dem Gewerbemüll entsprechender Müll mit Abfallstoffen aus Industriebetrieben; [bis hierhin nenne ich die Erläuerungen sachorientiert] oft muß seine Giftigkeit beachtet werden. (S. 104)

Der letzte Satz ist grundsätzlich problemorientiert zu nennen, auch wenn die Sehweise von Industriemüll als Problemthema nicht annähernd vollständig entfaltet wird.

Insgesamt sind die Lexika, ihrer zentralen Funktion zu informieren getreu, alle weitgehend sachorientiert. Nicht jedes Thema ist auch so strittig, daß es dazu eine ausgeprägte problemorientierte Sehweise gibt. Aber bei allen strittigen Themen käme es bei der Darstellung doch sehr darauf an, der oder den öffentlich kursierenden Sehweisen entsprechendes Gewicht zu verleihen. Genau dies geschieht bei den meisten der Lexika nicht, im Gegenteil: viele Darstellungen sind auf dem öffentlichen, problembewußten Auge völlig blind. Zum Beispiel Sondermüll bei Schwann. Der Text beginnt mit der zentralen Erklärung

der einem biologischen Abbau nicht unterliegende anorganische Abfall. Als S. werden in der Regel solche Stoffe bezeichnet, die sich von Hausmüll unterscheiden [usw.]. (S. 204)

Als Beispiele werden dann Krankenhausabfälle und radioaktiver Abfall genannt, als seien dies die einzigen oder die wichtigsten Arten von Sondermüll. In der öffentlichen Diskussion wird Sondermüll überwiegend im Zusammenhang mit grundwassergefährdenden Sondermülldeponien oder mit Protesten gegen den Bau von Sondermüllverbrennungsanlagen gebraucht. Die Darstellung des Schwann-Lexikons steht in keinerlei Zusammenhang mit dieser öfentlichen Diskussion, obwohl sie und die an ihr Beteiligten gerade der Anlaß zur Publikation solcher populären Nachschlagewerke waren. 
Ein weiteres Beispiel für eine alle öffentlich relevanten Aspekte eines Problemverhalts ausblendende Darstellung liefert ein neueres Lexikon (Schneider), das sich auf den ersten Blick durch eingestreute Sprüche alternativer Leitfiguren wie Frederic Vester und Häuptling Seattle und durch optische Aufmachung als ein Bekenntnisbuch der Ökologiebewegung ausgibt. Den Erläuterungstext zum Thema Gift würde man so, wie er dasteht, eher in einem medizinischen als in einem "grünen" Lexikon erwarten:

'Gift' nennt man jenen Stoff, der die Gesundheit schädigt, sei es nach kurzer oder auch nach fortdauernder Einwirkungszeit. Auch Stoffe, die uns harmlos erscheinen, können wie Gifte wirken, ... (S. 138f.)

Als Beispiele werden dann angeführt: Kochsalz, Alkohol, Nikotin, Medikamente und Zimmerpflanzen. Kein Hinweis indes auf Chemikalien, auf Gift in der Nahrung, in Shampoo oder Zahpasta usw. Alphabetisch folgt auf Gift das Stichwort Giftmüll mit einem Verweis auf Sondermüll und dann ein längerer, nur aus Telefonnummern bestehender Artikel zum Stichwort Giftnotrufzentralen. Alles, was zum Thema Gift erläutert wird, ist sachlich richtig, präsentiert aber eine Sehweise, in der Gift als Thema der Umweltdiskussion überhaupt nicht existiert. Ausblenden und Ignorieren sind Strategien jeder konträren Diskussion, also auch der Umweltdiskussion insgesamt. Problematisch wird es aber, wenn dies unter Ausnutzung der Objektivitätserwartung gegenüber lexikonartigen Texten geschieht.

Weitgehend problemorientiert und offen gegenüber den Sehweisen, die in der Umweltdiskussion real existieren, sind nur rororo und das Lexikon der Katalyse-Umweltgruppe (KiWi), wobei es dem letzteren ganz besonders darauf ankommt, sowohl in der Stichwortauswahl als auch in der Art der Darstellung keines der symptomfunktionalen Charakteristika wissenschaftlicher Seriosität und politischer Unparteilichkeit auszulassen. Die besondere Schwierigkeit für die Autoren besteht nun darin, daß sie sich mit dem entsprechend markierten Wortschatz und mit der sach- und technikorientierten Darstellung zugleich die Sehweise der Forschung und damit auch der Industrie einhandeln. ${ }^{21}$

21 Der Soziologe Ulrich Beck schreibt in einem Essay in Der Spiegel vom 29.2.1988, S. 200 - 201 uber die "Macht der Technik in der wissenschaftlichen Zivilisation": "Selbst ihre schärfsten Kritiker müssen die Sprache wechseln, also die Grundfehler, 
$\mathrm{Da}$ dieses Lexikon trotzdem zu den problemorientierten zu rechnen ist, liegt an der grundsätzlichen Bindung an die Sehweise einer Umweltgruppe, in der sachorientierte Erläuterungen nie isoliert dastehen. Zum Beispiel werden bei Abfallbeseitigungsplan (S. 4) und bei Umweltverträglichkeitsprüfung (S. 428) nicht nur Inhalt und Ziel dieser Regelungen dargestellt, sondern es wird auch erläutert, daß es sich dabei um unrealisierte, kaum wirksame und bisher unverbindliche Maßnahmen handelt, daß gewissermaßen die mit diesen Ausdrücken bezeichneten Gegenstände in der Welt nicht in gleicher Weise existieren wie andere Gegenstände, obwohl die Realexistenz suggeriert wird durch Angleichung an Zusammensetzungen wie $B e-$ bauungsplan, Landschaftsplan und Fahrprüfung, Rechnungsprüfung, Eignungsprüfung u.a.

Unter sprachlichem Aspekt könnte man Wörter wie Abfallplan und Umweltverträglichkeitsprüfung als "Phantomwörter" charakterisieren, denn sie lassen nicht erkennen, daß ihre Inhalte in die Kategorie 'Idee, Plan, statt; Konzept, Wunsch, Forderung, Ideal, Traum, Utopie' gehören. Eine rein sach- und administrationsorientierte Darstellung läßt den Aspekt des Unvollendeten oder nicht-Realen nicht deutlich werden; so erklärt Meyers Lexikon Umweltverträglichkeitsprüfung als ein "Verfahren zur Prüfung aller öfentlicher Maßnahmen ..." usw. und als "wichtiges Instrumentarium zur Durchsetzung des Vorsorgeprinzips" (S. 334). Das eine Phantomwort wird mittels eines weiteren Phantomworts, Vorsorgeprinzip, aufgewertet. Die Verwendung solcher Phantomwörter wären eine eigene Untersuchung wert. Mit der Art ihrer Erläuterung im Lexikon, soviel sollte nur gezeigt werden, läßt sich die Tragweite von problemorientierter und sachorientierter Themenbehandlung in sprachkritischer Hinsicht deutlich machen.

Gerade in der Umweltdiskussion, in der die Bezeichnungen Sachlichkeit, Objektivität, verständliche Angst der Bevölkerung und Irrationalität $\mathrm{zu}$ scheinargumentativen Wertprädikaten werden, geht die Bedeutung von Darstellungsstilen weit darüber hinaus, bloß "Stilfrage" zu sein. 


\section{Zusammenfassung}

Der Umgang mit Wörtern in Umweltlexika ist, bei einem so kontroversen Thema der öffentlichen Diskussion, wie es das Thema Umwelt ist, nicht anders als interessengeleitet möglich, auch dann, wenn der Text Objektivitätsanspruch erhebt und die Form einer Informationsinstanz annimmt. Die Interessegebundenheit resultiert nicht nur aus den politischen Standpunkten von Autoren und Verlag, sondern auch schon aus der Abhängigkeit von den Quellen, die ihrerseits schon vorher parteiisch und Meinungsäußerungen innerhalb der Diskussion waren.

Um Wörter in Übereinstimmung mit bestimmten Interessen zu verwenden und diese Verwendung den Lesern nahezulegen, setzen Lexikon-Autoren folgende Mittel ein:

1. Durch Festlegung der Stichwörter, Verweise und abgewerteten Bezeichnungsvarianten wird die Sehweise auf einen Problembereich in bestimmter Weise konstituiert und konstruiert.

2. Durch Aufwertung und Abwertung von Stichwörtern bzw. Bezeichnungsvarianten werden explizite Normierungsversuche unternommen und dabei mit pseudolinguistischen Argumenten begründet.

3. Durch überwiegende Sach- anstelle von Problemorientierung wird in den Erläuterungen eine Sehweise propagiert, die den Eindruck der Wissenschaftlichkeit erweckt und Wissenschaftlichkeit zur Stil- und Argumentationsnorm erhebt, aber ganz bestimmten politischen Zielen dient und tatsächlich vorhandene Aspekte eines Themas ausblendet.

4. Die Autorität und der Objektivitätsanspruch der Textsorte Lexikon wird gezielt zur Durchsetzung nicht-autoritativer und nichtobjektiver Meinungen benutzt. 
7. Anhang 


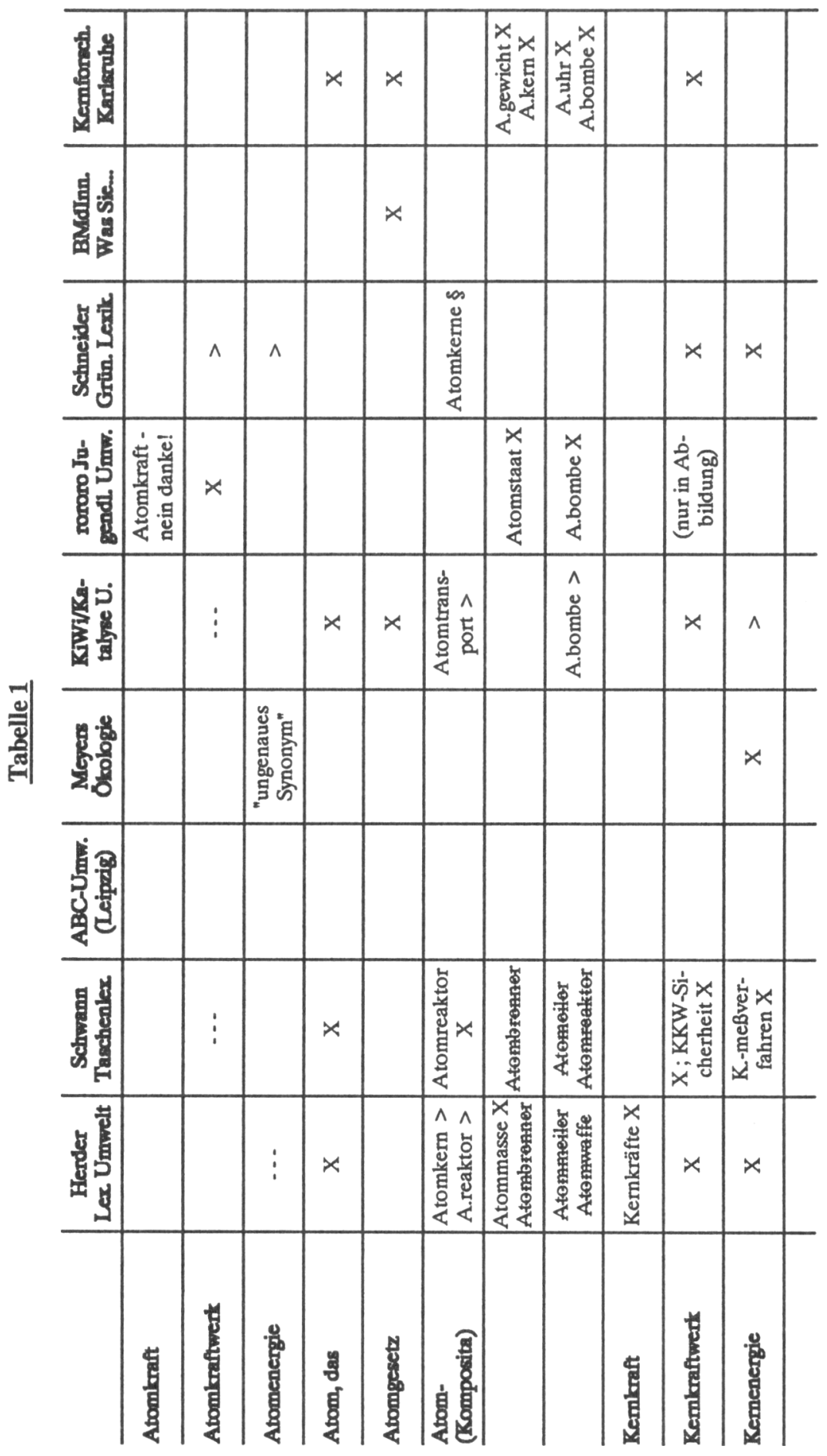




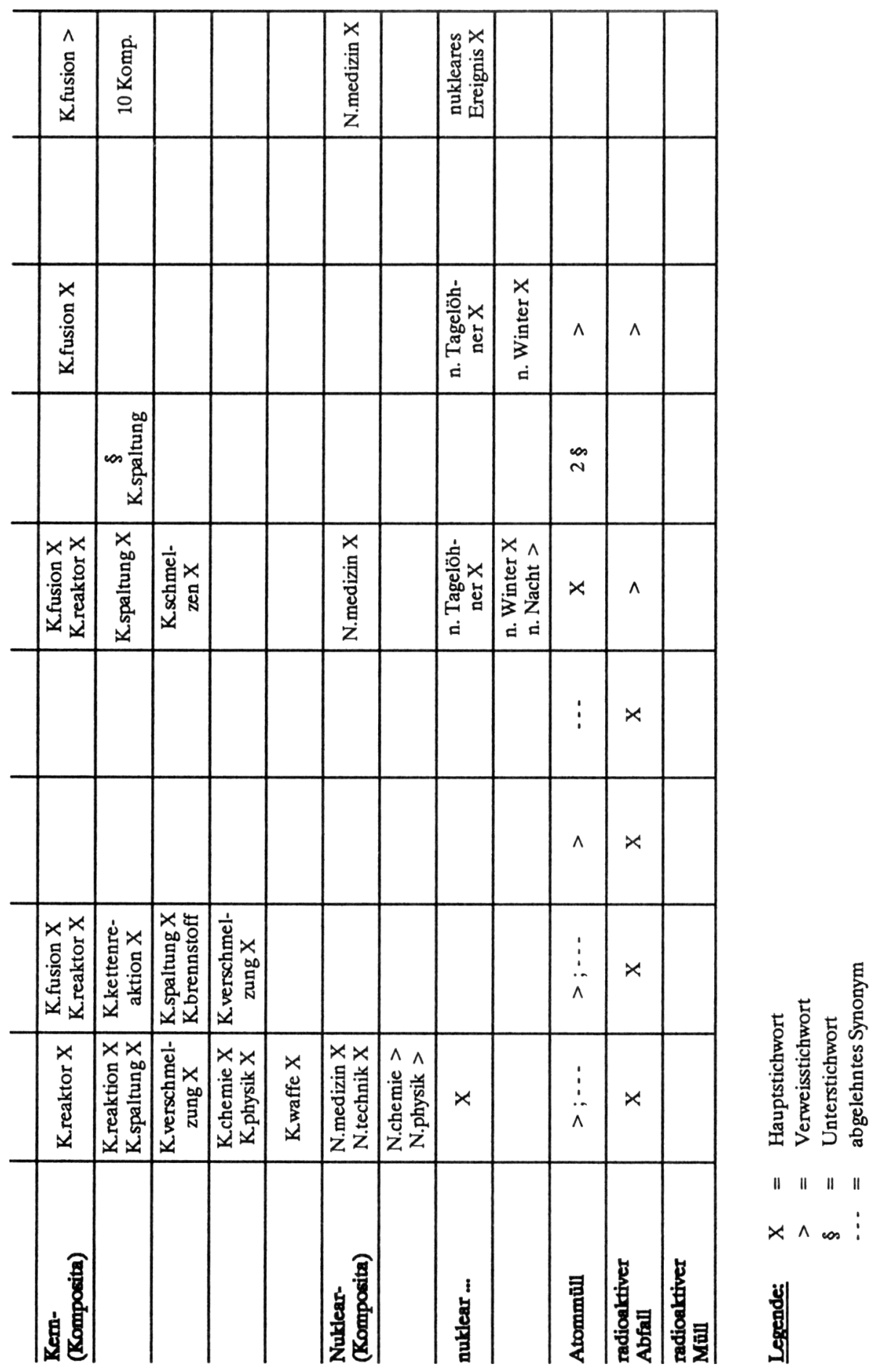




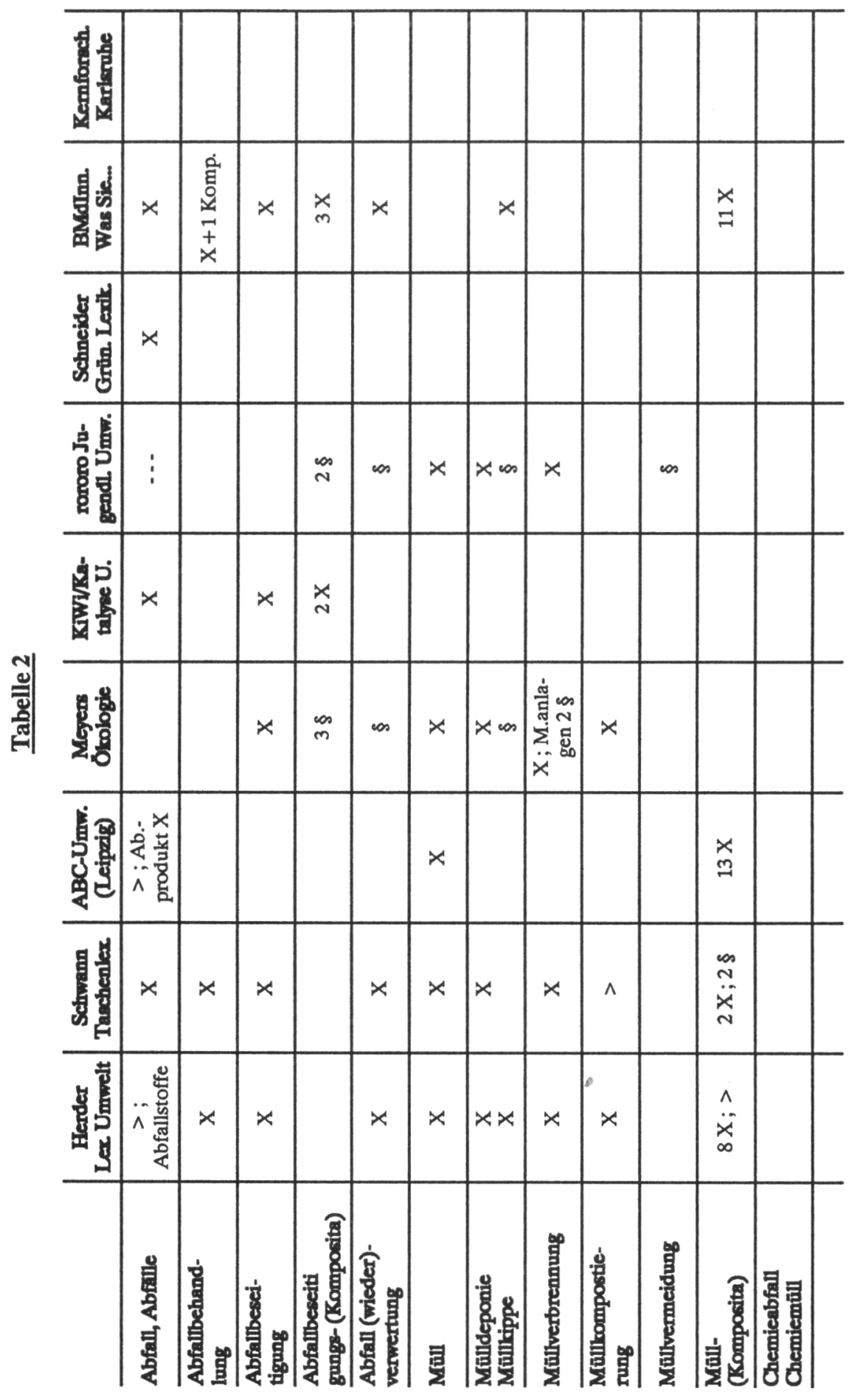




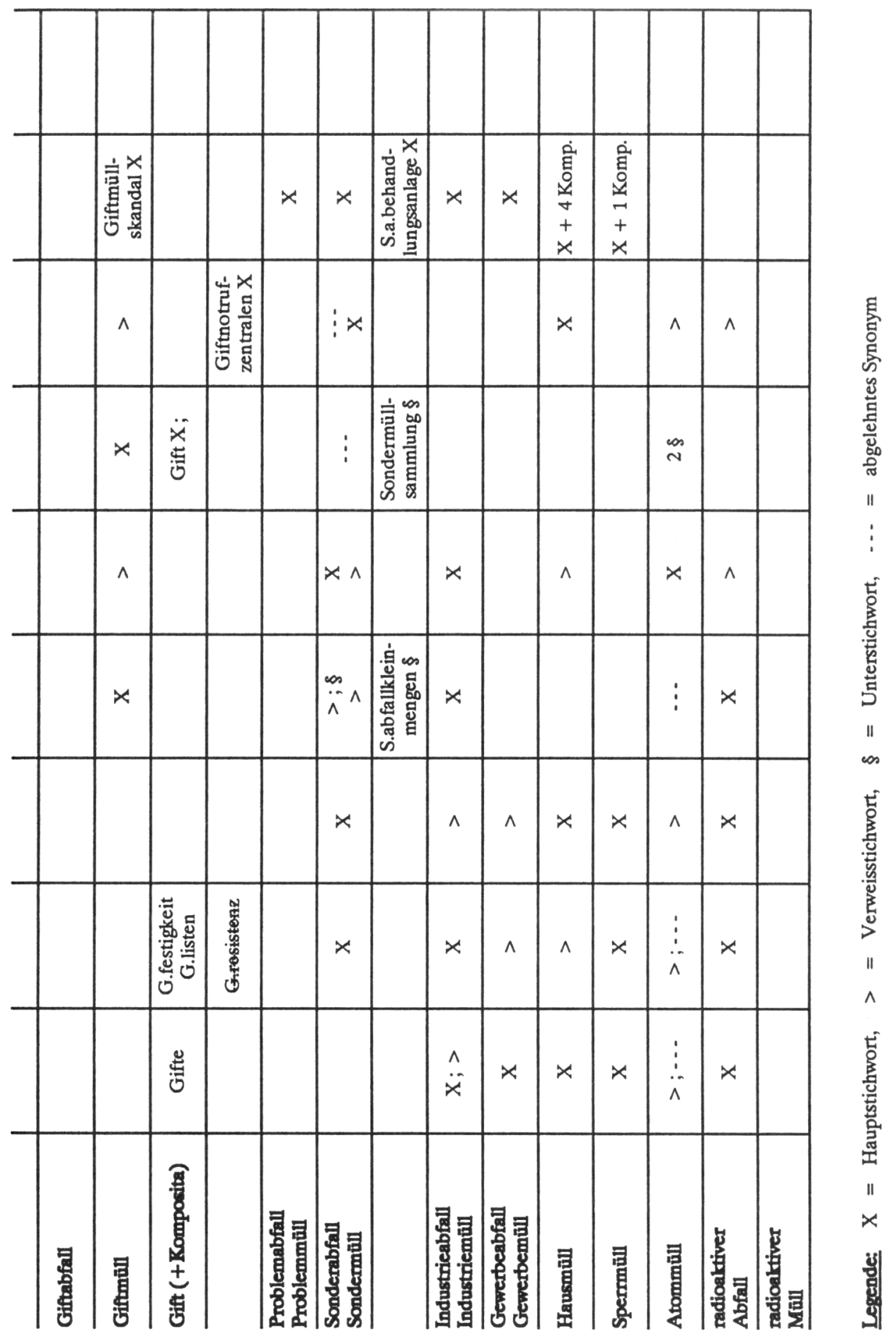




\section{Quellen}

HERDER LEXIKON Umwelt, bearb. im Auftrag der Lexikonredaktion von Udo Becker, 3. Auflage 1979 (1. A. 1973), Freiburg i.Br. ("mit rund 1800 Stichwörtern sowie über 300 Abbildungen und Tabellen", $215 \mathrm{~S}$.)

Otto Ahlhaus, Gerhard Boldt, Klaus Klein: TASCHENLEXIKON UMWELTSCHUTZ, Pädagogischer Verlag Schwann Düsseldorf, 5. Auflage 1981 (1. A. 1979) (288 S.)

ABC UMWELTSCHUTZ unter besonderer Berücksichtigung der Umweltschutztechnologie, hrsg. von der Arbeitsgruppe ABC Umweltschutz der Kommission Umweltschutz beim Präsidium der Kammer der Technik, VEB Deutscher Verlag für Grundstoffindustrie Leipzig, 3., überarbeitete und erweiterte Auflage, Leipzig 1984 (1. A. 1976) (Redaktionsschluß 31.1.1983) ("Mit 349 Bildern, 96 Tabellen und einem Anhang", 356 S.)

WAS SIE SCHON IMMER ÜBER UMWELTSCHUTZ WISSEN WOLLTEN, hrsg. v. Bundesministerium des Innern, Redaktion Karl G. Tempel, Volkardt Möcker, Kohlhammer Verlag, Stuttgart usw., 2. verbesserte u. erweiterte Aufl. 1984/85 (1. A. 1981) (312 S.)

WAS SIE SCHON IMMER ÜBER ABFALL UND UMWELT WISSEN WOLLTEN; hrsg. von s.0., 126.-135. Tausend: September 1984 (1. A. 1981) (227 S.)

Brunhilde Marquard, Helmut Mikelskis, Conrad Westhoff unter Mitarb. v. Karl-Hein Mau: JUGENDLEXIKON UMWELT. Umweltwissen in Stichworten, rororo, Reinbek, Dezember 1984 (341 S.)

UMWELT-LEXIKON. 2000 Stichworte aus den Gebieten ... hrsg. v.d. Katalyse-Umweltgruppe, Kiepenheuer \& Witsch, Köln 1985 (522 S.)

W. Kölzer; LEXIKON ZUR KERNENERGIE, hrsg. v. Kernforschungszentrum Karlsruhe $\mathrm{GmbH}$, Karlsruhe, 56.-16. Tausend Juni 1986 (1. A. 1980) (192 S.)

Michael und Edda Neumann-Adrian: DAS GRÜNE LEXIKON. Umwelt verstehen, Umwelt schützen. Mit e. Vorwort v. Frederic Vester, Franz Schneider, München 1986 (378 S.) 
MEYERS KLEINES LEXIKON ÖKOLOGIE. hrsg. u. bearb. v. Meyers Lexikonredaktion in Zus.arb. mit Prof. Dr. Klaus Wegmann, Bibliogr. Inst., Mannheim, 1987 (376 S.)

\section{Literatur}

Gülich, E. (1986): Textsorten in der Kommunikationspraxis. In: W. Kallmeyer (Hg.): Kommunikationstypologie. Jahrbuch 1985 des Instituts für deutsche Sprache (=Sprache der Gegenwart 67), Düsseldorf 1986, S. 15 - 46

Haß, U. (1986): Textsorten als Wirkungssystem. Zum popularisierenden Tranfer 'gelehrter' Inhalte in schriftlichen Texten an einem Beispiel aus dem 15./16. Jahrhundert. In: Deutsche Sprache, 3/1986, S. 224 - 234

Hermanns, F. (1982): Brisante Wörter. Zur lexikographischen Behandlung parteisprachlicher Wörter und Wendungen in Wörterbüchern der deutschen Gegenwartssprache. In: H.E. Wiegand (Hg.): Studien zur neuhochdeutschen Lexikographie II (=Germanistische Linguistik 3-6 / 1980), Hildesheim,Zürich/New York 1982, S. 87 - 108

Hermanns, F. (1986): Appellfunktion im Wörterbuch. Ein lexikographischer Versuch. In: H.E. Wiegand (Hg.): Studien zur neuhochdeutschen Lexikographie VI/1 (=Germanistische Linguistik 84-86 / 1986) Hildesheim, New York 1986, S. $151-182$

Höcker, K.H. (1957): Sollten Amerikanismen unübersetzt bleiben? Probleme der Begriffsbestimmung am Reaktor. In: Die Atomwirtschaft 11/1957, S. 373 - 375

Ischreyt, H. (1958): Die Sprache der Kernphysik und Kerntechnik. Versuch einer Bestandsaufnahme. In: Muttersprache $68 / 1958$, S. $65-72$

Kindlers Literaturgeschichte der Gegenwart Bd. $11=$ Die deutsche Sachliteratur III, R. Radler (Hg.), Frankfurt a.M. 1980

Küpper, H. (1982): Illustriertes Lexikon der deutschen Umgangssprache, 8 Bde., Stuttgart 1982-1984

Lötje, W. (1977): Der Einfluß der englischen Sprache auf die deutsche Militärsprache und auf die deutsche Allgemeinsprache und auf Denken und Verhalten Deutschsprachiger. In: Sprachspiegel 33/1977, S. 99 - 107 
Möhn, D. / Pelka, R. (1984): Fachsprachen. Eine Einführung. Tübingen 1984

Röthlein, B. (1979): Kernenergie, ein Thema der öffentlichen Meinung. Beschreibung und Analyse eines Kommunikationsprozesses. Diss. München 1979

Strauß, G. / Zinfonun, G. (1985): Die Semantik schwerer Wörter im Deutschen. Teil 1: Lexikologie schwerer Wörter (=Forschungsberichte des Instituts für deutsche Sprache Mannheim 58.1), Tübingen 1985

Strauß, G. (1986): Der politische Wortschatz. Zur Kommunikationsund Textsortenspezifik. (=Forschungsberichte des Instituts für deutsche Sprache Mannheim 60), Tübingen 1986 